\title{
Instrucción y entrenamiento: baluartes fundamentales para la seguridad marítima internacional
}

\author{
Álvaro Eduardo Díaz Rivera \\ Dirección, Escuela Internacional de Guardacostas, Cartagena, Colombia. \\ alvaro.diaz@armada.mil.co
}

Recibido: Septiembre 25, 2019.

Recibido en su versión corregida: Septiembre 25, 2019.

Aceptación: Octubre 24, 2019.

Cómo citar: Diaz Rivera, A.E. (2019). Instrucción y entrenamiento: baluartes fundamentales para la seguridad marítima internacional. Revista Sextante, 21, pp. 12 - 19, 2019.

\section{Resumen}

El narcotráfico es un fenómeno ilegal que afecta al entorno marítimo y todas sus actividades legales, tanto estatales como comerciales y privadas. Por su dinámica, complejidad y alcance requiere de una efectiva sinergia regional basada en la interoperabilidad entre los organismos estatales y las agencias de seguridad involucradas en la preservación de la seguridad y de la vida humana en el mar. El presente artículo pretende dar a conocer cómo la Escuela Internacional de Guardacostas, coadyuva a que la Armada Nacional de Colombia logre alcanzar esa sinergia, mediante la instrucción y entrenamiento de los hombres y mujeres que laboran en el mar; especialización que permite alcanzar la interoperabilidad planteada y se constituye en un arma valiosa para combatir las amenazas y actores ilegales en el mar, generando confianza y aumentado la cooperación internacional necesaria para lograr un entorno marítimo seguro y confiable.

Palabras clave: Amenaza; Entrenamiento; Interoperabilidad; Instrucción; Narcotráfico; Seguridad marítima; Sinergia; Transnacional.

\section{Instruction and training: Fundamental Bastions for International maritime security}

\begin{abstract}
Drug trafficking is an illegal phenomenon that affects the maritime environment and all its legal activities, both state and commercial and private. Due to its dynamics, complexity and scope, it requires an effective regional synergy based on interoperability between state agencies and security agencies involved in the preservation of security and human life at sea. This article aims to make known as the International Coast Guard School, helps the Colombian Navy achieve that synergy, through the instruction and training of men and women working in the sea; specialization that allows to achieve the interoperability raised and is constituted is a valuable weapon to combat threats and illegal actors at sea, generating trust and increasing the international cooperation necessary to achieve a safe and reliable maritime environment.
\end{abstract}

Keywords: Drug trafficking; Interoperability; Instruction; Maritime security; Synergy; Threat; Training; Transnational. 


\section{Transporte marítimo}

El transporte marítimo es el principal modo de realizar el comercio internacional. A diferencia del transporte aéreo, el de carretera o el ferroviario; la movilización marítima permite enviar grandes cantidades de mercancía a un costo muy económico (Pérez, 2012). En la Figura 1, un ejemplo de volumen de mercancía por unidad mercante. Es por ello por lo que para el actual paradigma globalista de las relaciones internacionales: transnacionalismo - interdependencia compleja, el escenario marítimo es el principal medio de interconexión comercial para todos los actores (estatales y privados) del sistema internacional.

Actualmente, alrededor del $90 \%$ del comercio mundial se realiza a través del transporte marítimo. Sin este no sería posible la importación y exportación de mercancías en la escala necesaria para mantener el mundo; su expansión constante se traduce en beneficios para todas las naciones.

Por lo anterior, los riesgos a los que se ve expuesto el transporte marítimo son enormes.

Todos los fenómenos ilícitos de naturaleza transnacional pueden afectar e incidir negativamente en todos los medios y modos de transporte en el mar.

Algunos de los mayores desafíos para la seguridad consisten en delitos que, si bien son cometidos en territorio nacional, trascienden las fronteras de los países y afectan a regiones enteras y en definitiva, a la comunidad internacional (ONU, 2013).

\section{Seguridad marítima}

De acuerdo con la Estrategia de Seguridad Marítima de la Unión Europea, se entiende por seguridad marítima una situación del sector marítimo mundial en la que se aplican el Derecho Internacional y las leyes nacionales. La libertad de navegación está garantizada y los ciudadanos, las infraestructuras, el transporte, el medio ambiente y los recursos marinos están protegidos (Información de la Estrategia de Seguridad Nacional y del Informe Anual de Seguridad Nacional, 2014).

Por ser de interés para toda la comunidad internacional, la seguridad marítima se convierte entonces en una prioridad para todos los Estados; los cuales, por la dinámica propia de la globalización, deben garantizar entornos marítimos jurisdiccionales seguros y coadyuvar para el mantenimiento de tales condiciones en las aguas internacionales.

De igual forma, las autoridades nacionales se ven abocadas a trabajar cada vez más, de manera combinada, interagencial e interinstitucional para garantizar la seguridad en las áreas marítimas y portuarias.

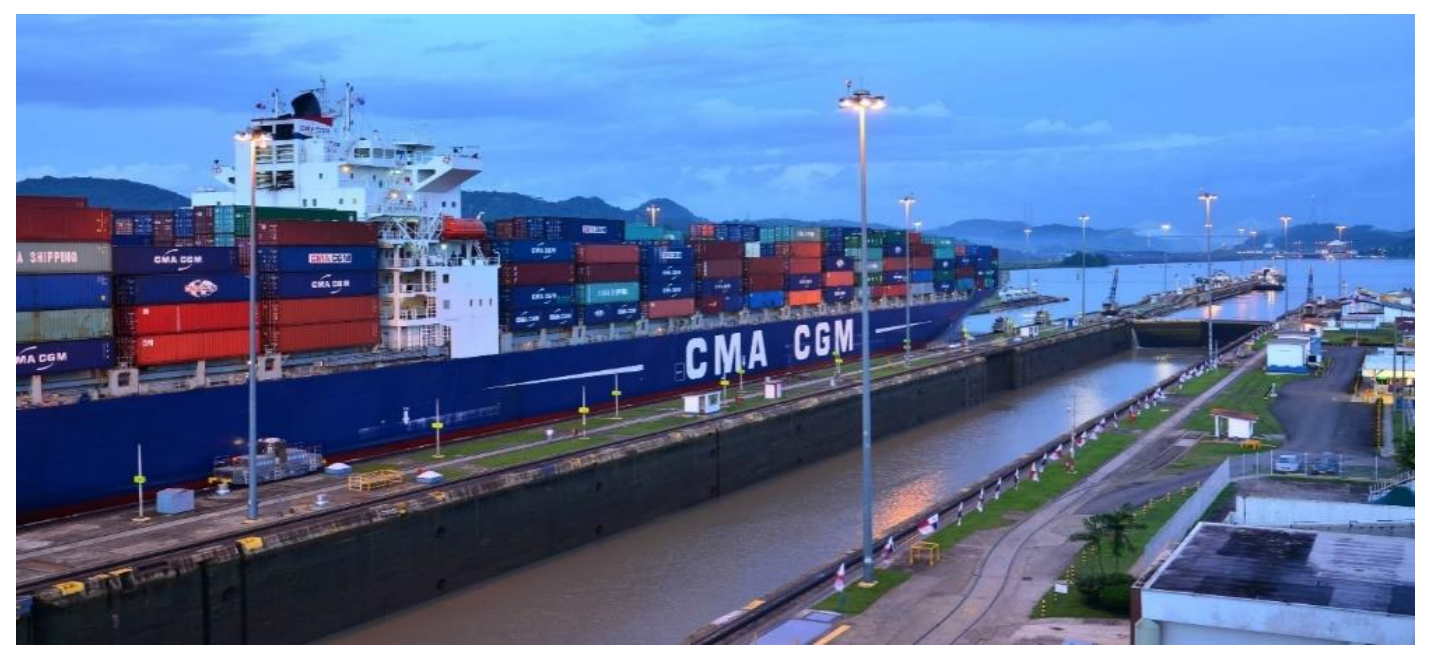

Figura 1. Transporte marítimo internacional.

Fuente: https://blogs.iadb.org/transporte/es/recesion-transporte-maritimo/ 


\section{La cooperación internacional en el entorno marítimo}

La cooperación internacional puede analizarse desde muchas aristas. En el ámbito de la seguridad marítima y enfocada hacia el interés común de un entorno marítimo seguro y confiable (en un ambiente con una pluralidad de actores); la cooperación internacional se compone por el tridente conceptual: capacitación - instrucción entrenamiento, el cual busca impactar positivamente el recurso más valioso de cualquier institución: el talento humano.

Recursos humanos - conocimiento - medios; son los bastiones fundamentales de cualquier marina de guerra, armada, fuerza naval, cuerpo de guardacostas y/u organismo marítimo de seguridad de cualquier estado nación en el mundo. El interés se centrará en los recursos humanos y en el conocimiento.

La experticia y destreza de sus integrantes, obtenidas a partir de una inversión constante en su instrucción y entrenamiento, se constituye en un arma poderosa y trascendental para cualquier institución estatal de seguridad marítima en el cumplimiento de su misión: combatir de manera efectiva y segura el uso ilícito de sus espacios marítimos jurisdiccionales, por parte de cualquier actor ilícito local o transnacional.

Es aquí donde la tarea silenciosa pero excepcional de las entidades dedicadas a la instrucción y entrenamiento de los miembros de las fuerzas de seguridad, en particular de las instituciones que se desenvuelven en el escenario marítimo; se torna eminentemente relevante para el mantenimiento de las condiciones necesarias para promover el desarrollo y el crecimiento de todos los países costeros alrededor del globo terráqueo.

\section{Seguridad marítima y cooperación internacional}

La transnacionalidad del crimen organizado es un problema que atañe a todos los actores (estatales, públicos y privados) intervinientes en el escenario marítimo.

Los gobiernos están caracterizando como desafíos de seguridad a aquellos problemas antes considerados como cuestiones policiales, e inclusive amenazas a la sanidad pública. A diferencia de las amenazas tradicionales orientadas hacia el Estado, las amenazas transnacionales muchas veces surgen de una forma gradual y comúnmente resulta difícil precisar sus causas y efectos (Vásquez, 2006)

Migración ilegal, piratería, narcotráfico y contrabando, entre otras; son las principales amenazas a la seguridad marítima.

\section{Crimen transnacional}

El crimen organizado transnacional (Figura 2): desde las redes criminales hasta los grupos insurgentes y organizaciones terroristas, tienen un hilo común: el dinero (Mavrellis, 2017).

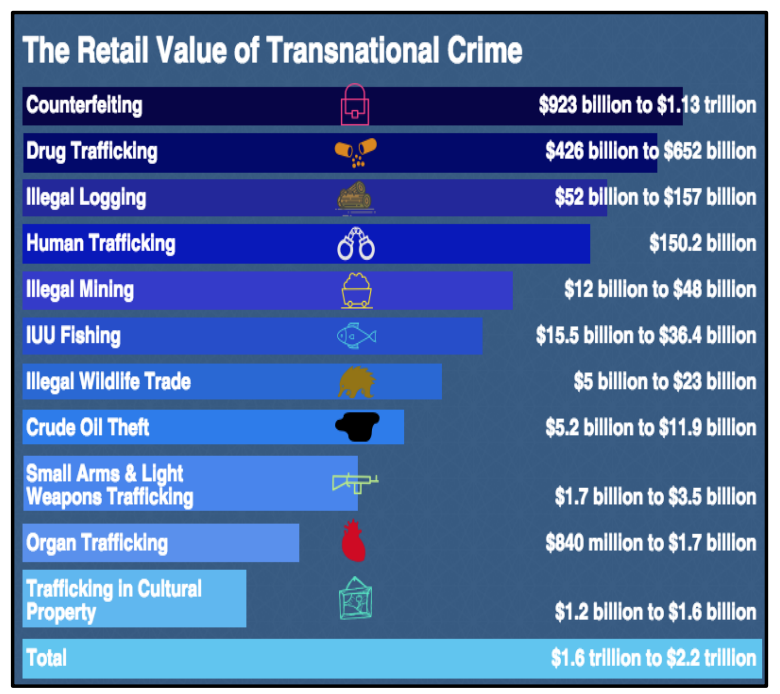

Figura 2. El valor minorista de la delincuencia transnacional. Fuente: https://gfintegrity.org/business-transnational-crime/

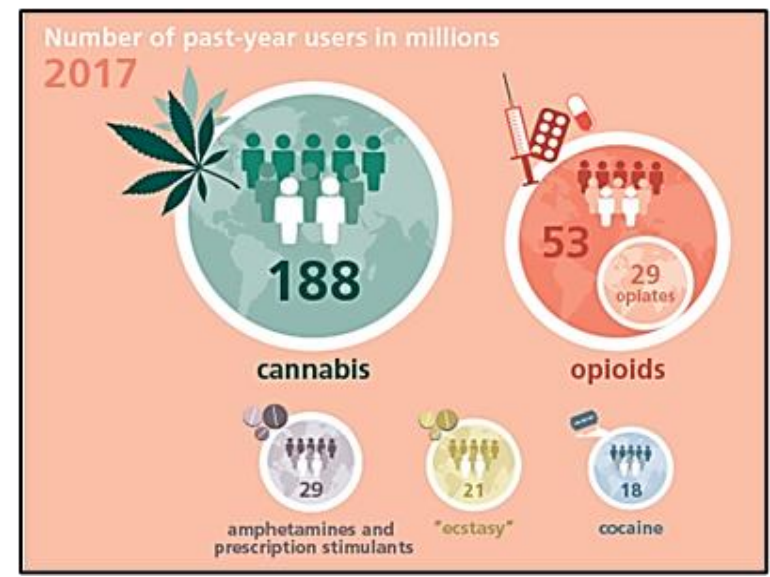

Figura 3. Número de consumidores en unidad de millón del año 2017.

Fuente:

http://www.odc.gov.co/Portals/1/publicaciones/pdf/internacio nales/WDR19_Booklet_2_DRUG_DEMAND.pdf 
Sin ocupar el primer lugar del ranking, el narcotráfico es la amenaza que genera un gran impacto y afectación en el transporte marítimo y por ende, en la seguridad marítima. Según la Oficina de las Naciones Unidas Contra la Droga y el Delito (en adelante: UNODC por su sigla en inglés), existen más de trecientos millones de personas que consumen sustancias ilícitas en el mundo. En la Figura 3, ejemplo de las sustancias ilícitas con mayor demanda.

\section{La Escuela Internacional de Guardacostas y la seguridad marítima}

La Escuela Internacional de Guardacostas (en adelante: ESCUIG) es un centro de formación y capacitación de carácter internacional, que capacita, entrena y evalúa a oficiales, suboficiales e infantes de marina profesionales, en funciones y actividades propias de guardacostas. Lo anterior, para propender por el máximo nivel de alistamiento de las tripulaciones mediante la aplicación de las normas, leyes, procedimientos y normatividad vigente; con el propósito de contribuir al cumplimiento de la misión institucional de las autoridades marítimas.

Bajo la premisa anterior, la ESCUIG entrena y capacita al personal de la Armada Nacional de Colombia (en adelante: ARC) en procedimientos de interdicción marítima, de abordaje, visita y registro, de búsqueda y rescate y de supervivencia en el mar, bajo la estricta aplicación de la ley y las normas procedimentales en el mar; con el fin de garantizar la seguridad en las aguas jurisdiccionales del país.

Mediante la instrucción y el entrenamiento, la ESCUIG coadyuva directamente para preservar la vida humana en el mar: función principal de cualquier ente de guardacostas.

La ESCUIG, por la relevancia de su función institucional, contribuye activamente en la lucha contra las amenazas transnacionales en el escenario marítimo (específicamente en la lucha contra el fenómeno ilegal del narcotráfico marítimo y fluvial); mediante la transmisión de conocimientos a través de talento humano idóneo y experimentado, así como con el empleo de recursos tecnológicos. Por lo tanto, la ESCUIG crea, planea e implementa programas de capacitación encaminados a suministrar los conocimientos apropiados para el desarrollo de las operaciones propias del cuerpo de guardacostas; con el propósito de contribuir a la seguridad y defensa de la nación.

Actualmente su portafolio de servicios consta de dieciséis cursos de carácter teórico - práctico en la modalidad 'Escuela' y cuatro cursos en la modalidad 'Grupo Móvil de Entrenamiento' (en adelante: GME).

Mediante el desarrollo de asignaturas teóricoprácticas - que incluyen nociones básicas de Derechos Humanos y de Derecho Internacional Humanitario - los alumnos de los cursos de la ESCUIG (en ambas modalidades) reciben los conocimientos necesarios para salvaguardar la vida humana y contrarrestar las amenazas transnacionales en el mar (particularmente el fenómeno del narcotráfico).

\section{La Escuela Internacional de Guardacostas y la cooperación internacional}

La Escuela de Guardacostas, unidad orgánica de la ARC, está ubicada en la ciudad de Cartagena de Indias (D.T. y C.) en Colombia. Fue creada el 06 de noviembre de 2009 y a partir del 11 de septiembre de 2015 se convirtió en la ESCUIG. Su función es entrenar y capacitar al personal de oficiales, suboficiales e infantes de marina profesionales de la ARC; así como a los miembros de las armadas, marinas, fuerzas navales y cuerpos de guardacostas; en dos misiones trascendentales del rol propio de los cuerpos de guardacostas e instituciones equivalentes en el mundo: la lucha contra los fenómenos y las redes ilegales transnacionales, así como la preservación de la vida humana en el mar.

La ESCUIG es un centro de formación y capacitación de gente de mar, de carácter regional; el cual capacita, entrena y evalúa a oficiales, suboficiales e infantes de marina profesionales en funciones y actividades propias de guardacostas. Lo anterior, para propender por el máximo nivel de alistamiento de las tripulaciones, mediante la aplicación de las normas, leyes, procedimientos y normatividad vigente; con el propósito de contribuir al cumplimiento de la misión institucional de las autoridades marítimas.

En pro de lo anterior, la ESCUIG entrena, capacita y reentrena al personal de las instituciones 
militares y civiles análogas a la ARC; en abordaje e interdicción marítima, supervivencia en el mar, búsqueda y rescate; bajo los parámetros y las normas procedimentales internacionales, con el fin de coadyuvar al mantenimiento de la seguridad en el escenario marítimo continental.

La ESCUIG cuenta con personal orgánico con amplia experiencia, con la experticia y competencias reglamentarias para la instrucción y capacitación del personal de alumnos nacionales e internacionales. Es por ello que su contribución en la lucha contra las amenazas transnacionales en el mar es determinante para la materialización y obtención de resultados operacionales tangibles e intangibles por parte de los organismos e instituciones comprometidas con la seguridad de los espacios marítimos; condición indispensable para el crecimiento y desarrollo de cualquier país en el mundo. En dichos términos, la labor de la ESCUIG es altamente relevante por su alcance e impacto regional y continental.

Es por ello por lo que la ESCUIG, al propender por la materialización de una "sinergia regional a través de la interoperabilidad", contribuye al fortalecimiento de los lazos de cooperación internacional.

\section{Instrucción y entrenamiento}

Desde el año 2009 y hasta la fecha, la ESCUIG ha capacitado y entrenado un total de 5.261 tripulantes a través de su oferta académica.

En la modalidad 'Escuela' se han entrenado y capacitado 2.097 personas en la ESCUIG. Así mismo, han sido entrenados y capacitados 1.204 mediante la modalidad 'GME' nacional (Ver Figura 4).

El 'Curso Integral de Guardacostas' (modalidad 'Escuela') ha sido impartido a 673 tripulantes de la ARC, para que puedan desempeñarse en las unidades operativas del cuerpo de guardacostas de la ARC (ver Figura 5).

De igual forma, la ESCUIG está comprometida con el nuevo modelo de educación de la ARC (en adelante: MEDAR), el cual entró en vigor a partir de enero de 2019; mediante el cual se han entrenado y capacitado hasta el momento a 88 Marineros
Segundos de la ARC, a través del 'Curso Integral de Guardacostas', relación que se muestra en la Figura 6.

Así mismo, otro de los dieciséis cursos en la modalidad 'Escuela', es el de Interdicción Marítima Internacional, el cual se realiza en las instalaciones de la ESCUIG (ver Figura 7 y $\underline{8}$ ). Dicha capacitación la han realizado 423 estudiantes (148 alumnos nacionales colombianos y 275 alumnos extranjeros de 25 países del mundo de tres continentes).

En adición a lo anterior, la ESCUIG lleva a cabo entrenamientos en la modalidad 'GME' en todo el continente americano. De la Figura 9, se muestra la relación de 766 tripulantes capacitados de seis países de Centroamérica y el Caribe.

Cabe resaltar, que recientemente la ESCUIG incorporó a su oferta académica el 'Curso Internacional de Interdicción Marítima Avanzado', con el objetivo de brindar las competencias necesarias para planear y liderar operaciones de interdicción marítima relacionadas con el combate de amenazas transnacionales (puntualmente contra el tráfico de sustancias ilícitas por vía marítima) y operaciones de búsqueda y rescate; integrando el uso de medios de superficie y aeronavales.

El primer curso se desarrolló con una cohorte de diez oficiales alumnos: 04 de Colombia, 01 de Guatemala, 03 de Honduras y 02 de República Dominicana; con el apoyo de la Escuela Naval de Cadetes "Almirante Padilla" de la ARC y la Escuela de Aviación Naval de la ARC.

\section{Sinergia a través de la interoperabilidad}

El funcionamiento de la ESCUIG en sus instalaciones actuales se logró concretar gracias al apoyo de la Oficina Internacional de Asuntos de Antinarcóticos y aplicación de la Ley (en adelante: INL por su sigla en inglés), dependencia del Departamento de Estado de los Estados Unidos.

Por otra parte, la ESCUIG hace parte del Plan de Acción Conjunto de Colombia y Estados Unidos para Centroamérica y El Caribe (en adelante: USCAP por su sigla en inglés) del Comando Sur de los Estados Unidos, el cual es un esfuerzo colaborativo de cooperación en seguridad que está orientado en el establecimiento y el mejoramiento 


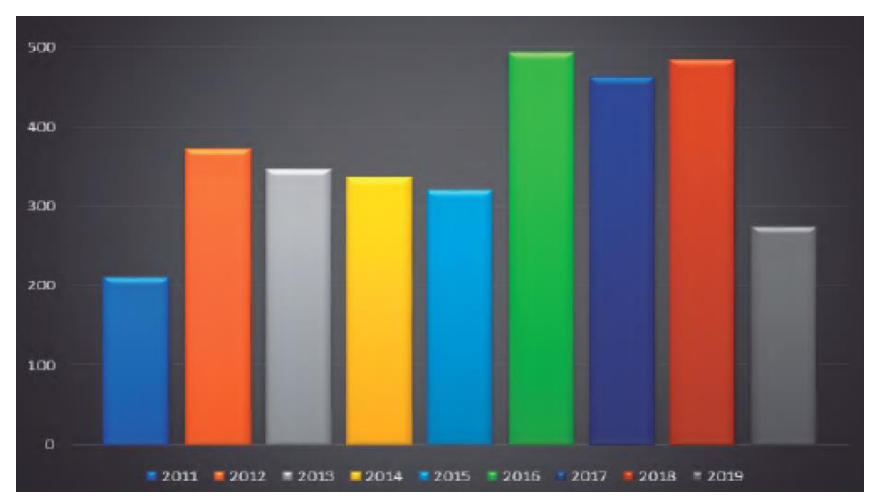

Figura 4. Capacitación nacional ESCUIG.

Fuente: Los autores.

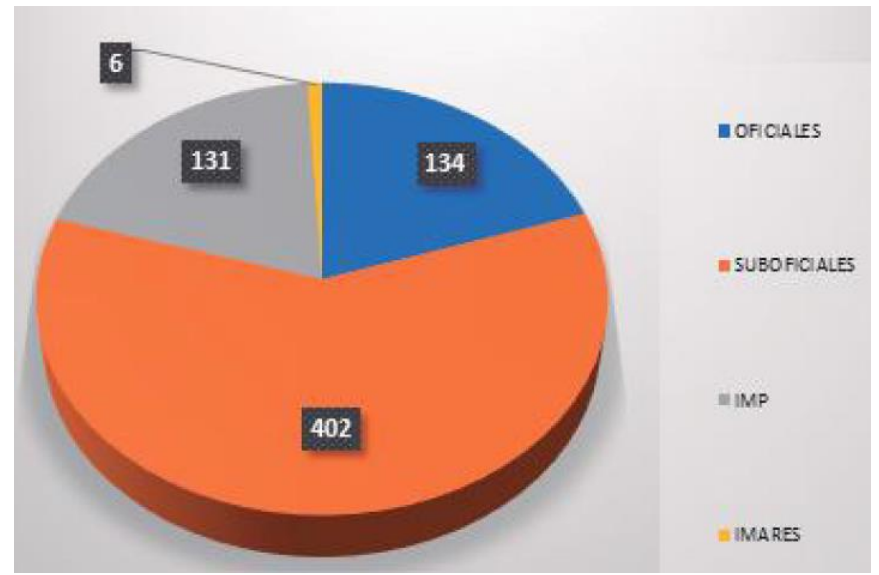

Figura 5. Relación de integrantes del curso integral de guardacostas ESCUIG.

Fuente: Los autores.

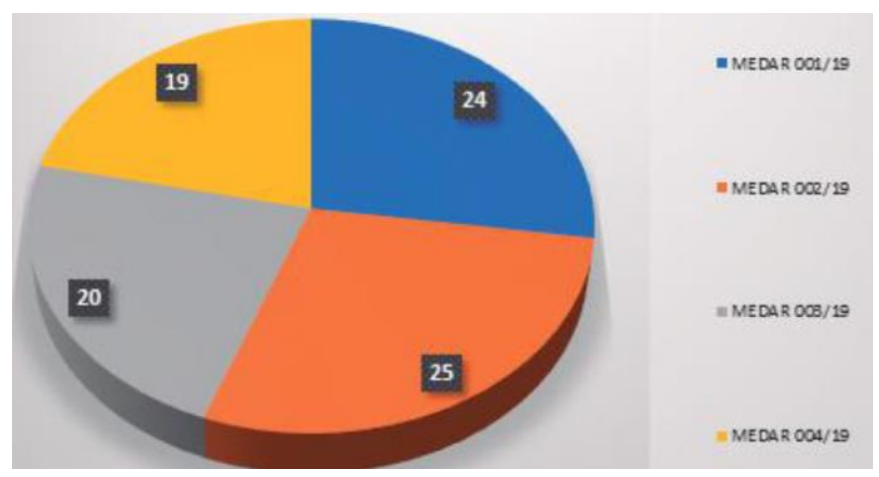

Figura 6. Curso integral guardacostas MEDAR ESCUIG.

Fuente: Los autores.

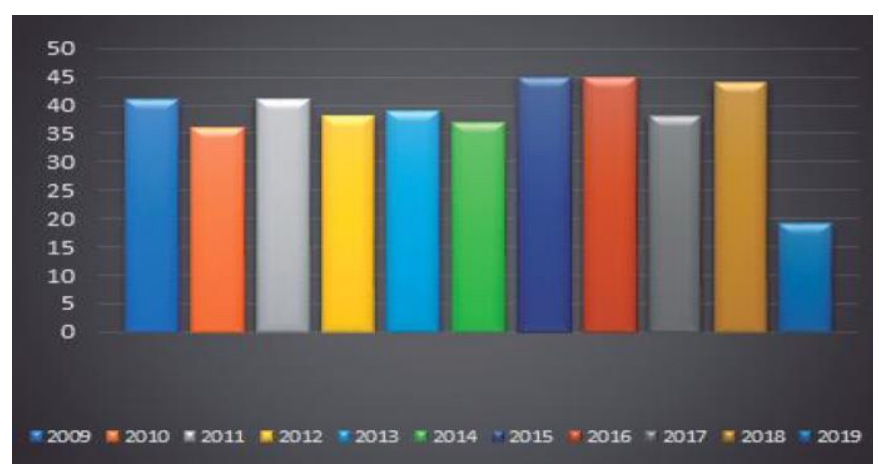

Figura 7. Curso internacional de interdicción marítima ESCUIG.

Fuente: Los autores.

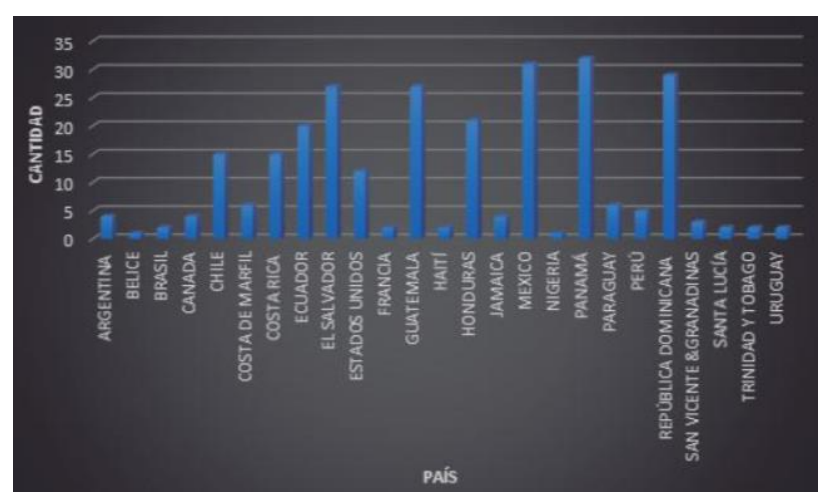

Figura 8. Nacionalidades de los alumnos extranjeros del curso internacional de interdicción marítima ESCUIG.

Fuente: Los autores.

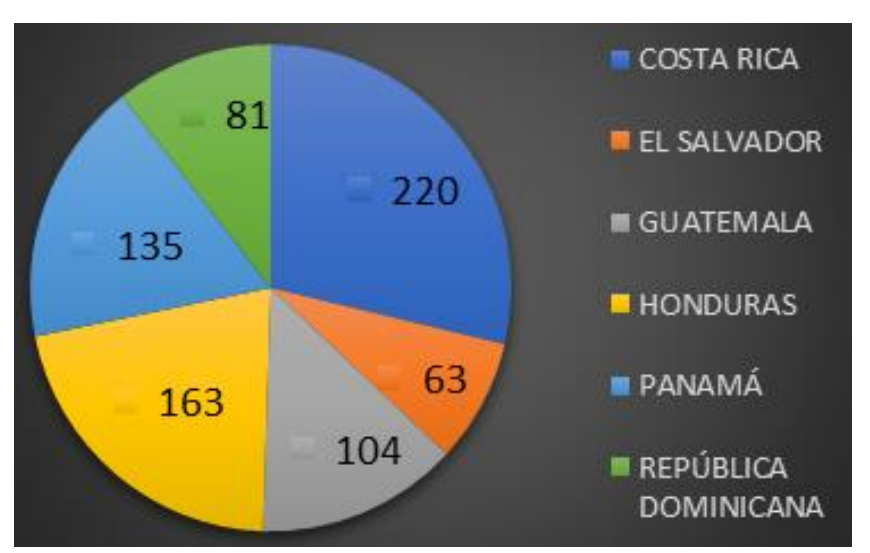

Figura 9. GME internacional ESCUIG.

Fuente: Los autores.

de las capacidades regionales para contrarrestar amenazas transnacionales.

Tanto USCAP como INL patrocinan y apoyan alumnos nacionales e internacionales, en varios cursos de la modalidad 'Escuela' de la ESCUIG, así como entrenamientos en el exterior (modalidad 'GME').

Con base en ello, la visión de largo plazo de la ESCUIG contribuye activamente, no solo a la estrategia de la ARC para combatir el tráfico de narcóticos y sus delitos conexos materializados por parte de organizaciones criminales transnacionales (en adelante: OCT), sino también en la proyección institucional de la ARC como una marina mediana de proyección regional.

La ESCUIG tiene como meta convertirse en un referente a nivel internacional en la instrucción, el entrenamiento y la capacitación de los miembros de las instituciones navales, organismos de seguridad, entes intervinientes en el control y seguridad del entorno marítimo, inclusive en la gente de mar. 
La amenaza del narcotráfico es compleja, dinámica y adaptativa; por ello se requiere de esfuerzos y acciones combinadas, interagenciales e interinstitucionales para contrarrestar su accionar delictivo. Esto es lo que se denomina sinergia y su materialización se logra mediante el entrenamiento y capacitación del talento humano en el mar.

\section{Narcotráfico: amenaza emergente y no tradicional (pero no nueva)}

El narcotráfico no es un fenómeno ilegal producto de los "desajustes" de la modernidad. Es una actividad transnacional histórica. Data del siglo XIX con el tráfico del opio desde la India hacia Francia e Inglaterra (su comercialización en China generó las “Guerras del Opio"). La globalización le ha servido para diversificarse y hacer más eficiente su metodología, con el fin de reducir los márgenes de riesgo y aumentar las probabilidades de éxito.

En el siglo pasado, en la década de los setenta, fue cuando se empezó a combatir el narcotráfico por vía marítima, como un problema de salud y/o de seguridad.

Actualmente, el narcotráfico reúne todas las condiciones para ser una verdadera amenaza mundial de naturaleza emergente (nuevos y múltiples actores) y no tradicional (de carácter asimétrico e irregular).

\section{Multisectorial y multidimensional}

Su naturaleza es diversa por la facultad de poder afectar los cuatro campos del poder (políticoeconómico-social e inclusive el militar); es multidimensional debido a que se desenvuelve en todos los escenarios: marítimo, aéreo y terrestre.

\section{Impacta la seguridad y la estabilidad}

Tiene la capacidad de afectar la seguridad (impactar negativamente a todos los actores del sistema internacional, tanto públicos como privados) y la estabilidad (impactar negativamente a las sociedades) en todos los países del mundo.

\section{Trasciende las fronteras}

Es un flagelo global debido al gran alcance de su rango de acción. El narcotráfico demanda de esfuerzos y acciones unificadas para contrarrestarlo.

\section{La profundidad de la amenaza del narcotráfico}

El narcotráfico tiene una relación intrínseca con ambos tipos o clases de amenazas. En el caso de las amenazas "duras", es un constituyente primario, debido a que promulga e impulsa la ocurrencia de estas. En el caso de las amenazas "blandas", está íntimamente relacionado con los indicadores (educación, empleo/ingresos, salud, servicios públicos y vivienda) de las necesidades básicas insatisfechas - desigualdad social.

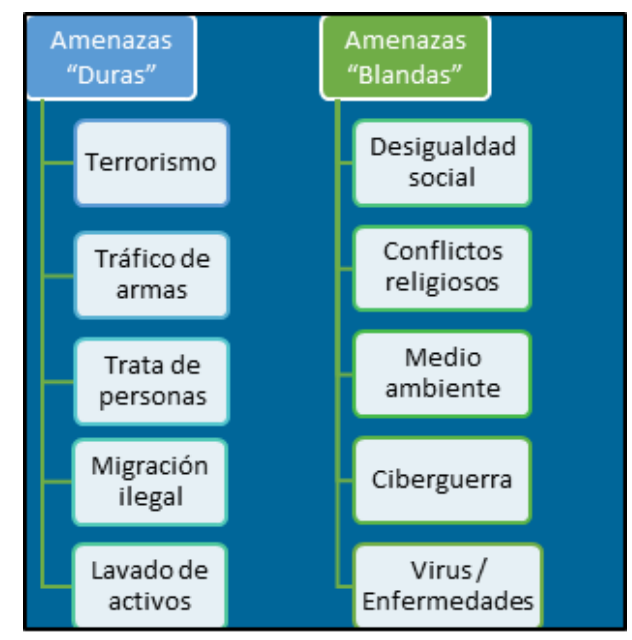

Figura 10. Clases de amenazas.

Fuente: Los autores. (Bénitez \& García, 2007).

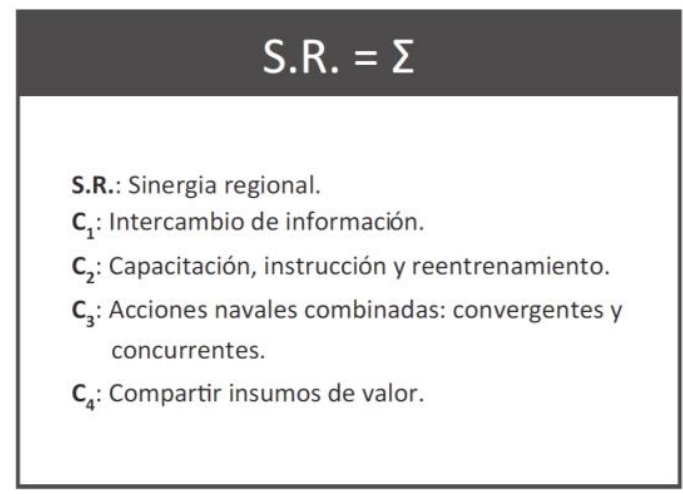

Figura 11. Sinergia regional a través de la interoperabilidad. Fuente: Los autores. 


\section{2. ¿Qué hacer?}

El narcotráfico es un desafío tanto para la seguridad del transporte marítimo y todas sus actividades legales, como para la estatalidad y la legalidad. Su complejidad hace que sea difícil luchar contra él. Además, su dinámica (en constante evolución e innovación) lo hace fuerte y su capacidad de adaptación a la actuación de los organismos de seguridad le permite tener alcances de nivel global.

Es por ello por lo que la sinergia para contrarrestar su accionar delictivo e ilegal, se puede lograr a partir de la conjugación de cuatro elementos esenciales: Colaboración (C1) - Cooperación (C2) - Coordinación (C3) - Confianza (C4).

Esta denominada "Sinergia Regional" tiene en uno de sus componentes (C2) la participación y acción directa de la Escuela Internacional de Guardacostas de la Armada Nacional de Colombia (ver Figura 11); como institución encargada de lograr materializar la interoperabilidad entre las instituciones y organizaciones intervinientes en la seguridad del escenario marítimo; a través de la instrucción, entrenamiento y capacitación de los hombres y mujeres que laboran diariamente en el mar.

\section{Referencias}

Bénitez, M., \& García, M. (2007). Annan, Los Objetivos del Milenio: las amenazas duras versus las amenazas blandas. Kofi. Cuascatlán: Universidad centroamericána José Simeón Cañas.

Información de la Estrategia de Seguridad Nacional y del Informe Anual de Seguridad Nacional. (19 de enero de 2014). Departamento de Seguridad Nacional. Obtenido de Seguridad Marítima: https://www.dsn.gob.es/es/sistemaseguridad-nacional/qu\%C3\%A9-es-seguridadnacional/\%C3\%Almbitos-seguridadnacional/seguridad-mar\%C3\%ADtima

Mavrellis, C. (11 de abril de 2017). GFI. Obtenido de The Business of Transnational Crime: https://gfintegrity.org/business-transnationalcrimel
ONU. (27 de noviembre de 2013). La ONU y el ESTADO DE DERECHO. Obtenido de Amenazas transnacionales: https://www.un.org/ruleoflaw/es/thematicareas/transnational-threats/

Organización Marítima Internacional. (20 de 11 de 2015). OMI. Obtenido de "El transporte marítimo: indispensable para el mundo", seleccionado como lema del Día marítimo mundial de 2016: http://www.imo.org/es/MediaCentre/PressBriefi ngs/Paginas/47-WMD-theme-2016-.asp

Perez, J. (2012 de diciembre de 2012). El transporte marítimo. Recuperado el 2018 de octubre de 15

Vásquez, J. (2006). Amenazas Translacionales. Rioja: Boletín de información. 